\title{
Theorems connected with the Differentiation of a Circulant.
}

\author{
By Professor W. H. Metzler. \\ (Read and Received 9th May 1919).
}

1. In 1909 Dr Thomas Muir, ${ }^{*}$ in a paper on the above topic, gave several theorems involving the derivation of a circulant, and it is the writer's purpose in this paper to extend these investigations with a number of other results.

2. Let the imaginary factors of the circulant $C$ be denoted by $\alpha_{1}, \alpha_{2}, \ldots, \alpha_{2 m}$, where $\alpha_{k}=a_{1}+a_{2} \theta^{k}+a_{3} \theta^{2 k}+\ldots+a_{n} \theta^{(n-1) k}$, and taking their products in pairs as follows: $\alpha_{1}, \alpha_{2 m}, \alpha_{2}, \alpha_{2 m-1}$, $\ldots \alpha_{m}, \alpha_{m+1}$, let them be denoted by $\alpha_{1,2 m}, \alpha_{2}, \alpha_{2 m-1}, \ldots \alpha_{m, m+1}$ respectively. Let us denote the sum

$$
\sum_{1}^{m_{k}} \alpha_{1,2 m} \cdot \alpha_{2,2 m-1} \ldots \alpha_{k, 2 m-k+1} \text { by } \sum_{1}^{m_{k}} \alpha_{j}^{(k)}
$$

then it is readily seen that

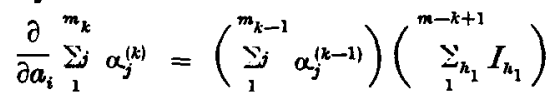

where $I_{h_{1}}$ represents $\left(\alpha_{n_{1}} \theta^{m-h_{1}}{ }^{(i-1)}+\alpha_{2 n+1-h_{1}} \theta^{h_{1}(i-1)}\right)$, and where for each $\alpha_{j}^{(k-1)}$ in $\Sigma_{j} \alpha_{j}^{(k-1)}$, the co-factor $\Sigma I_{h_{1}}$ contains no $\alpha$ which is found in $x_{j}^{(k-1)}$.

Again,

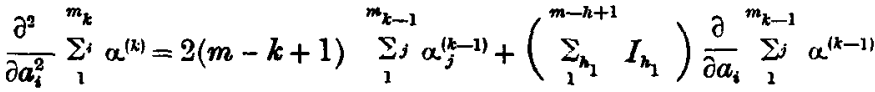

$$
\begin{aligned}
& =2(m-k+1) \sum_{1}^{m_{k-1}} \alpha_{j}^{(k-1)}+2\left(\sum_{1}^{m_{k-2}} \alpha_{j}^{(k-2)}\right)^{(m-k+2)_{2}} \sum_{1}^{\sum} I_{h_{1}} I_{h_{2}}
\end{aligned}
$$

where in each term of $\Sigma \alpha_{j}^{(k-2)}$ there is no $\alpha$ common to $\alpha_{j}^{(k-2)}$, $I_{h_{1}}$, and $I_{h_{2}}$.

"Muir, Messenger of Maths., Now Series, No. 460, August 1909. 
In general,

(R)

$$
\begin{aligned}
\frac{\partial^{r}}{\partial a_{i}^{r}} \sum_{i}^{m_{k}} \alpha_{j}^{(k)}=2(r-1)(m+ & k+1) \frac{\partial^{r-2}}{\partial a_{i}^{r-2}} \stackrel{m_{k-1}}{\sum_{j} \alpha_{j}^{(k-1)}} \\
& +\left(\begin{array}{ll}
m-k+1 \\
\sum_{h_{1}} & I_{h_{1}}
\end{array}\right) \frac{\partial^{r-1}}{\partial a_{i}^{r-1}}\left(\begin{array}{c}
m_{k-1} \\
\sum_{j} \alpha_{j}^{(k-1)}
\end{array}\right)
\end{aligned}
$$

Taking the sum with respect to $i$ in each case, we have

(A') $\sum_{1}^{n} \frac{\partial}{\partial a^{i}} \sum_{1}^{m_{k}} \alpha_{j}^{(k)}=0$

(B') $\sum_{1}^{n} \frac{\partial^{2}}{\partial a_{i}^{2}} \sum_{1}^{m_{k}} \alpha_{j}^{(k)}=2 \cdot n \cdot(m-k+1) \sum_{1}^{m_{k-1}} \alpha_{j}^{(k-1)}$

(R') $\sum_{1}^{n} \frac{\partial^{r}}{\partial a_{i}^{r}} \sum_{1}^{m_{k}} a_{j}^{(k)}=2(r-1)(m-k+1) \sum_{1}^{n} \frac{\partial^{r-2}}{\partial a_{i}^{r-2}} \sum_{1}^{m_{k-1}} \alpha_{j}^{(k-1)}$

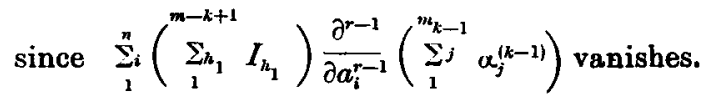

From ( $\mathrm{R}^{\prime}$ ) we have, when $r=2 p$,

$$
\begin{aligned}
\sum_{1}^{n} \frac{\partial^{2 p}}{\partial a_{i}^{2 p}} \sum_{1}^{m_{k}} \alpha_{j}^{(k)} & =2 .(2 p-1)(m-k+1) \stackrel{1}{1}_{1}^{n} \frac{\partial^{2 p-2}}{\partial a_{i}^{2 p-2}}\left(\sum_{1}^{m_{k-1}} \alpha_{j}^{(k-1)}\right) \\
& =2^{p} \cdot n \cdot(m-k+1)(m-k+2) \ldots(m-k+p) \\
& \times(2 p-1)(2 p-3) \ldots 3.1 . \sum_{1}^{\sum_{k-k}} \alpha_{j}^{(k-p)},
\end{aligned}
$$

and when $r=2 p+1$

(2) $\quad \stackrel{\aleph}{\Sigma}_{i} \frac{\partial^{2 p+1}}{\partial a_{i}^{p+1}} \stackrel{m_{k}}{\Sigma_{j}} \alpha_{j}^{(k)}=0$.

3. It is known* that

$$
\text { for } n=2 m+1
$$

I. $\sum_{1}^{m_{k}} \alpha_{j}^{(k)}=(-1)^{k} \Sigma\left(\begin{array}{l}12 \ldots 2 k \\ 12 \ldots 2 k\end{array}\right),(k=1,2, \ldots m)$,

II. $S . \sum_{1}^{m_{k}} \alpha_{j}^{(k)}=(-1)^{k} \Sigma\left(\begin{array}{c}12 \ldots 2 k+1 \\ 12 \ldots 2 k+1\end{array}\right),(k=0,1, \ldots m)$;

$$
\text { and for } n=2 m+2
$$

- Vide author's paper in the Mathematics Teacher, December 1918. 
III. $\quad S . S^{\prime} \sum_{1}^{m_{k-1}} \alpha_{j}^{(k-1)}-\sum_{1}^{m_{k}} \alpha_{j}^{(k)}{ }^{*}=(-1)^{k-1} \Sigma\left(\begin{array}{l}12 \ldots 2 k \\ 12 \ldots 2 k\end{array}\right)$, $(k=1,2, \ldots m+1)$,

IV. $\left(S+S^{\prime}\right) \sum_{j}^{m_{k}} \alpha_{j}^{(k)}=(-1)^{k} \Sigma\left(\begin{array}{l}12 \ldots 2 k+1 \\ 12 \ldots 2 k+1\end{array}\right),(k=0,1, \ldots, m)$

$$
\text { where } \begin{aligned}
S & =a_{1}+a_{2}+a_{3}+\ldots, \\
S^{\prime \prime} & =a_{1}-a_{2}+a_{3}-\ldots,
\end{aligned}
$$

and where $\Sigma\left(\begin{array}{l}12 \ldots k \\ 12 \ldots k\end{array}\right)$, (or $\Sigma_{k}$ say), is the sum of the coaxial minors of order $k$.

4. For circulants of odd order, $n=2 m+1$, we have from (1) and $I$

$$
\text { (3) } \begin{aligned}
\sum_{1}^{n} i \frac{\partial^{2 p}}{\partial a_{i}^{2 p}} \Sigma_{2 k}= & -2 \cdot(2 p-1)(m-k+1) \stackrel{n}{\Sigma_{1}} \frac{\partial^{2 p-2}}{\partial a_{i}^{2 p-2}} \Sigma_{2 k-2} \\
= & (-1)^{p} 2^{i p} \cdot n \cdot(2 p-1)(2 p-3) \ldots 3 \cdot 1 \cdot(m-k+1) \\
& \cdots(m-k+p) \Sigma_{2 k-2 p} .
\end{aligned}
$$

For $p=1$ and $k=m$, this is

$$
\begin{aligned}
\sum_{1}^{n} \frac{\partial^{2}}{\partial a_{i}^{2}} \Sigma A_{11} & =-2 n \Sigma_{2 m-2} \\
& =-\frac{2 n \Sigma_{2 n-1}}{S} .
\end{aligned}
$$

From (2) and I we have

(4) $\sum_{1}^{n} \frac{\partial^{2 p+1}}{\partial a_{i}^{2 p+1}} \Sigma_{2 k}=0$, which, for $p=1$ and $k=m$, is

$$
\sum_{1}^{n} \frac{\partial}{\partial a_{i}} \Sigma A_{11}=0 \text {. }
$$

From the relation $S . \Sigma_{2 k}=\Sigma_{2 k+1}$, we have

$$
\begin{aligned}
& \text { (A') } \frac{\partial}{\partial a_{i}} \Sigma_{2 k+1}=\Sigma_{2 k}+S \cdot \frac{\partial}{\partial a_{i}} \Sigma_{2 k} \\
& \text { (B") } \frac{\partial^{2}}{\partial a_{i}^{2}} \Sigma_{2 k+1}=2 \frac{\partial}{\partial a_{i}} \Sigma_{2 k}+S \frac{\partial^{2}}{\partial a_{i}} \Sigma_{2 k} .
\end{aligned}
$$

- It is to be observed that in this relation, when $k=m+1$, we must consider $\Sigma i \alpha_{j}^{(m+1)}$ to be zero. 
$\left(\mathrm{R}^{\prime \prime}\right) \quad \frac{\partial^{r}}{\partial a_{i}^{r}} \Sigma_{2 k+1}=r \frac{\partial^{r-1}}{\partial a_{i}^{r-1}} \Sigma_{2 k}+S \frac{\partial^{r}}{\partial a_{i}^{r}} \Sigma_{2 k}$.

From $\left(\mathrm{R}^{\prime \prime}\right)$ we have, when $r=2 p$,

(5)

$$
\begin{aligned}
\sum_{1}^{n} \frac{\partial^{2 p}}{\partial a_{i}^{2 p}} \Sigma_{2 k+1} & =S . \sum_{1}^{n} \frac{\partial^{2 p}}{\partial a_{i}^{2 p}} \Sigma_{2 k} \\
& =(-1)^{p} 2^{p} \cdot n . S \cdot(2 p-1)(2 p-3) \ldots 3.1 . \\
& \times(m-k+1)(m-k+2) \ldots(m-k+p) \Sigma_{2 k-2 p},
\end{aligned}
$$

which, for $p=1$ and $k=m$, gives

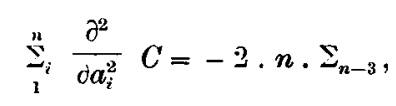

but $\quad \sum_{1}^{n} \frac{\partial^{2}}{\partial a_{i}^{2}} C=n \stackrel{\Sigma}{n} i_{1}^{n} \frac{\partial}{\partial \alpha_{i}} A_{i}, *$

$$
\therefore \quad \sum_{i} \frac{\partial A_{i}}{\partial a_{i}}=-2 \Sigma_{n-3} .
$$

When $r=2 p+1$ we have

$$
\begin{aligned}
\sum_{1}^{n} \frac{\partial^{2 p+1}}{\partial a_{i}^{2 p+1}} \Sigma_{2 k+1}= & (2 p+1) \sum_{1}^{n} \frac{\partial^{2 p}}{\partial a_{i}^{2 p}} \Sigma_{2 k} \\
= & (-1)^{p} \cdot 2^{p} \cdot n \cdot(2 p+1)(2 p-1)(2 p-3) \ldots 3.1 \\
& \times(m-k+1)(m-k+2) \ldots(m-k+p) \Sigma_{2 k-2 p},
\end{aligned}
$$

which, for $p=0$ and $k=m$, gives

$$
\sum_{1}^{n} i \frac{\partial}{\partial a_{i}} C=n \Sigma_{s m}=n \Sigma A_{1} \dagger
$$

where $A_{k}$ is the signed complementary minor corresponding to the element $a_{k}$ in the first row of $C$.

Since

it follows that

$$
\frac{\partial}{\partial a_{i}} \Sigma_{2 k+1}=S \cdot \frac{\partial}{\partial a_{i}} \Sigma_{2 k}+\Sigma_{n k} \text { and } \frac{\partial}{\partial a_{j}} \Sigma_{2 k+1}=S \cdot \frac{\partial}{\partial a_{j}} \Sigma_{2 k}+\Sigma_{2 k}
$$

(7) $\frac{\partial}{\partial a_{i}} \Sigma_{2 k+1}-\frac{\partial}{\partial a_{j}} \Sigma_{2 k+1}=S\left(\frac{\partial}{\partial a_{i}} \Sigma_{2 k}-\frac{\partial}{\partial a_{j}} \Sigma_{2 k}\right)$, 
or when $k=m$,

$$
\begin{aligned}
& \quad n\left(A_{i}-A_{j}\right)=S\left(\frac{\partial}{\partial a_{i}} \Sigma A_{11}-\frac{\hat{\partial}}{\partial a_{j}} \sum A_{11}\right) \\
& \text { since } \frac{\partial}{\partial a_{i}} C=n A_{i} .
\end{aligned}
$$

But by Stern's theorem *

$$
A_{i}-A_{j}=(-1)^{i+j-1} S \cdot Q
$$

where $Q$ is the determinant got from $C$ by deleting the first row and the $i^{\text {th }}$ and $j^{\text {th }}$ columns and inserting a column of units in the first place.

\section{Therefore}

$$
\frac{\partial}{\partial a_{i}} \sum A_{11}-\frac{\partial}{\partial a_{j}} \Sigma A_{11}=(-1)^{i+j-1} \cdot n \cdot Q .
$$

5. For circulants of even order, $n=2 m+2$, we have from IV.

$$
\begin{aligned}
& \left(\mathrm{A}^{\prime \prime \prime}\right) \quad(-1)^{k} \frac{\partial}{\partial a_{i}} \Sigma_{2 k+1}=\left(1+(-1)^{i-1}\right) \sum_{1}^{\sum_{k}} \alpha_{j}^{(k)}+\left(S+S^{\prime \prime}\right) \frac{\partial}{\partial a_{i}} \sum_{1}^{m_{k}} \alpha_{j}^{(k)} \\
& \left(\mathrm{B}^{\prime \prime \prime}\right) \quad(-1)^{k} \frac{\partial_{2}}{\partial a_{i}^{2}} \Sigma_{2 k+1}=2\left(1+(-1)^{i-1}\right) \frac{\partial}{\partial a_{i}} \sum_{1}^{m_{k}} \alpha_{j}^{(k)}+\left(S+S^{\prime}\right) \frac{\partial^{2}}{\partial a_{i}^{2}} \sum_{1}^{m_{k}} \alpha_{j}^{(k f} \\
& \left(\mathrm{R}^{\prime \prime \prime}\right) \quad(-1)^{k} \frac{\partial^{r}}{\partial a_{i}^{r}} \Sigma_{2 k+1}=r\left(1+(-1)^{i-1}\right) \frac{\partial^{r-1}}{\partial a_{i}^{r-1}} \sum_{1}^{m_{k}} \alpha_{j}^{(k)}
\end{aligned}
$$

$$
+\left(S+S^{\prime}\right) \frac{\partial^{r}}{\partial \alpha_{i}^{r}} \sum_{i}^{m_{i}} \alpha_{j}^{(k)}
$$

From $\left(\mathrm{R}^{\prime \prime \prime}\right)$ we have, when $r=2 p$

$$
\begin{aligned}
\sum_{1}^{n} \frac{\partial^{2 p}}{\partial a_{i}^{2 p}} \sum_{2 k+1}= & (-1)^{k}\left(S+S^{\prime}\right) \sum_{1}^{n} \frac{\partial^{2 p}}{\partial a_{i}^{2 p}} \sum_{1}^{m_{k}} \alpha_{j}^{(k)} \\
= & (-1)^{p} \cdot 2^{p} \cdot n \cdot(2 p-1)(2 p-3) \ldots 3.1 \\
& \times(m-k+1)(m-k+2) \ldots(m-k+p) \sum_{2 k-2 p+1},
\end{aligned}
$$

which, for $p=1$ and $k=m$, is

$$
\sum_{1}^{n} \frac{\partial^{2}}{\partial a_{i}^{2}} \Sigma A_{11}=-2 n \Sigma_{2 m-1}=-2 n \Sigma_{n-3} \text {. }
$$

*Crelle's Journal, lxxii., pp. 374-380. Cf. Muir, Mess. Math., New Series, No. 491, March 1912. 
When $r=2 p+1$, we have

(10) $\sum_{1}^{n} i \frac{\partial^{2 p+1}}{\partial a_{i}^{2 p+1}} \Sigma_{2 k+1}=(-1)^{k}(2 p+1) \sum_{1}^{n}\left(1+(-1)^{i}\right) \frac{\partial^{2 p}}{\partial a_{i}^{2 p}} \sum_{1}^{m_{k}} \alpha_{j}^{(k)}$

$$
\begin{aligned}
&=(-1)^{k+p} 2^{p} \cdot n \cdot(2 p+1)(2 p-1) \ldots 3.1 . \\
& \\
& \quad \times(m-k+1)(m-k+2) \ldots \\
&(m-k+p) \frac{\Sigma_{2 k-2 p+1}}{\Sigma a_{11}} .
\end{aligned}
$$

which, when $p=0$ and $k=m$ is

From III. we have

$$
\sum_{1}^{n} \frac{\partial}{\partial a_{i}} \sum A_{11}=n \frac{\sum A_{11}}{\Sigma a_{11}}
$$

$$
\begin{aligned}
& \text { (A } \left.\mathrm{A}^{\mathrm{iv}}\right)(-1)^{k-1} \frac{\partial}{\partial a_{i}} \mathrm{\Sigma}_{2 k}=\left(S^{\prime \prime}+(-1)^{i-1} S\right) \sum_{1}^{m_{k-1}} \alpha_{j}^{(k-1)} \\
& \times S . S^{\prime \prime} \frac{\partial}{\partial a_{i}} \sum_{1}^{m_{k-1}} \alpha_{j}^{(k-1)}-\frac{\partial}{\partial a_{i}}{\stackrel{1}{m_{k}}}_{\sum_{j}}^{m_{j}} \alpha_{j}^{(k)} \\
& \left(\mathrm{B}^{17}\right) \quad(-1)^{k-1} \frac{\partial^{2}}{\partial a_{i}^{2}} \Sigma_{2 k}=2\left(S^{\prime \prime}+(-1)^{i-1} S\right) \frac{\partial}{\partial a_{i}} \Sigma \alpha_{j}^{(k-1)} \\
& \times S . S^{\prime \prime} \frac{\partial^{2}}{\partial a_{i}^{2}} \Sigma \alpha_{j}^{(k-1)}-\frac{\partial^{2}}{\partial a_{i}^{2}} \Sigma \alpha_{j}^{(k)}+2(-1)^{b-1} \Sigma \alpha_{j}^{(k-1)} \\
& \left(\mathbf{R}^{\mathrm{iv}}\right) \quad(-1)^{k-1} \frac{\partial^{r}}{\partial a_{i}^{r}} \Sigma^{2 \omega}=r\left(S^{H}+(-1)^{i} S\right) \frac{\partial^{r-1}}{\partial a_{i}^{r-1}} \Sigma \alpha_{j}^{(k-1)}+S \cdot S^{\prime} \frac{\partial^{r}}{\partial a_{i}^{r}} \Sigma \alpha_{j}^{(k-1)} \\
& -\frac{\partial^{r}}{\partial a_{i}^{r}} \Sigma \alpha_{j}^{(k)}+r(r-1)(-1)^{i} \frac{\partial^{r-2}}{\partial a_{i}^{r-2}} \Sigma \alpha_{j}^{(k-1)} .
\end{aligned}
$$

From $\left(\mathrm{R}^{1 v}\right)$ we have when $r=2 p$

$$
\begin{aligned}
&(-1)^{k-1} \sum_{i}^{n} \frac{\partial^{2 p}}{\partial a_{i}^{2 p}} \Sigma_{2 k}=2 p \sum_{1}^{n}\left(S^{\prime \prime}+(-1)^{i} S\right) \frac{\partial^{2 p-1}}{\partial a_{i}^{2 p-1}} \Sigma_{\alpha_{j}^{(k-1)}} \\
&+\sum_{1}^{n} S \cdot S^{\prime \prime} \frac{\partial^{2 p}}{\partial a_{i}^{2 p}} \Sigma_{\alpha_{j}^{(k-1)}}-\sum_{1}^{n} \frac{\partial^{2 p}}{\partial a_{i}^{2 p}} \Sigma_{\alpha_{j}^{(k)}} \\
&+ 2 p(2 p-1) \sum_{1}^{n}(-1)^{i} \frac{\partial^{2 p-2}}{\partial a_{i}^{2 p-2}} \Sigma_{\alpha_{j}^{(k-1}} \\
&=(-1)^{k-p} 2^{p} \cdot n \cdot(2 p-1)(2 p-3) \ldots \\
& 3.1 \cdot(m-k+2) \ldots(m-k+p) \\
& \times\left\{p \frac{\Sigma_{2 k-2 p+1}}{\Sigma a_{11}}-(m-k+p+1) \Sigma_{2 k-2 p}\right\}
\end{aligned}
$$


which, when $p=1$ and $k=m+1$, is

$$
\sum_{1}^{n} \frac{\partial^{2}}{\partial a_{i}} C=-2 n \Sigma_{2 m-1}=-2 n \Sigma_{n-3} .
$$

When $r=2 p+1$ we have

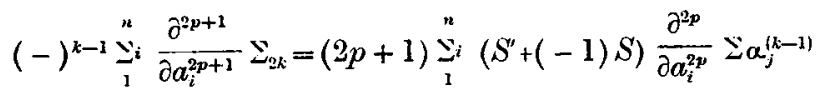

$$
\begin{aligned}
& +\sum_{1}^{n} S . S^{\prime} \frac{\partial^{2 p+1}}{\partial a_{i}^{2 p+1}} \sum \alpha_{i}^{(k-1)}-\sum_{1}^{n} \frac{\partial^{2 p+1}}{\partial a_{i}^{2 p+1}} \sum \alpha_{j}^{(k)} \\
& +(2 p+1) 2 p \sum_{i}^{n}(-1)^{i} \frac{\partial^{2 p-1}}{\partial a_{i}^{2 p-1}} \sum \alpha_{j}^{(i)} \\
& =(-1)^{k-p-1} 2^{p} \cdot n \cdot S^{\prime} \cdot(2 p+1)(2 p-1) \ldots \\
& \text { 3. } 1 \text {. }(m-k+2) \ldots \\
& (m-k+p+1) \frac{\Sigma_{2 k-2 p+1}}{\Sigma a_{11}}
\end{aligned}
$$

which, when $p=0$, and $k=m+1$, is

when $p=0$ and $k=1$, it is

$$
\Sigma \frac{\partial}{\partial a_{i}} C=\frac{n \cdot \omega^{\prime} \Sigma A_{11}}{\Sigma a_{11}}=n \Sigma A_{1}
$$

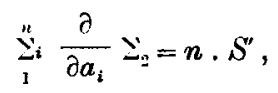

when $p=1$ and $k=m+1$, it is

$$
\begin{aligned}
& \stackrel{\aleph}{v}_{i} \frac{\bar{\sigma}^{2}}{\partial a_{i}^{2}} C=2 n\left(\frac{\Sigma_{2 m+1}-\Sigma a_{11} \Sigma_{2 m}}{\Sigma a_{11}}\right) \\
& =-2 n \frac{S \cdot S^{\prime \prime}}{\sum a_{11}} \cdot \aleph_{2 m-1}=-2 n \frac{S \cdot S^{\prime \prime}}{S^{\prime \prime} S^{\prime \prime}} \cdot \nu_{n-3},
\end{aligned}
$$

but

$$
\begin{aligned}
& \stackrel{n}{\Sigma}_{i} \frac{\hat{\sigma}^{2}}{\partial a_{i}^{2}} C=n \stackrel{\aleph_{i}}{\Sigma_{3}} \frac{\partial}{\partial a_{i}} A_{i} . \\
& \therefore \quad \sum_{i}^{n} \frac{\partial A_{i}}{\partial a_{i}}=-2 \cdot \frac{S \cdot S^{\prime}}{S+S^{\prime \prime}} \Sigma_{n-1},
\end{aligned}
$$

6. The relations given without proof in this article are either well known or are readily obtained from those which are.

$$
\frac{\partial C}{\partial a_{i}}=n A_{i} ; \quad \frac{\partial C}{\partial a_{i}}=n A_{j},
$$


$\therefore \frac{\partial A_{i}}{\partial a_{j}}=\frac{\partial A_{j}}{\partial a_{i}}, \sum_{1}^{n} \frac{\partial C}{\partial a_{i}}=n \sum_{1}^{n} A_{i}=n \Sigma A_{i}$ and $\sum_{1}^{n} \frac{\partial^{2} C}{\partial a_{i}^{2}}=n \sum_{1}^{n} \frac{\partial}{\partial a_{i}} A_{l}$.

Since $C=\Sigma a_{1} \Sigma A_{1}$ and $\frac{\partial C}{\partial a_{i}}=\Sigma A_{1}+\Sigma a\left(\frac{\partial}{\partial a_{i}} \Sigma A_{1}\right)$,

$$
\therefore \quad \frac{\partial}{\partial a_{i}} \Sigma A_{1}=\frac{n A_{i}-\Sigma A_{1}}{\Sigma a_{1}} \text { and } \Sigma \frac{\partial}{\partial a_{i}} \Sigma A_{1}=0 \text {. }
$$

Since $\quad C=\alpha_{1}, \alpha_{2} \ldots \alpha_{n}$

$$
\begin{aligned}
& \frac{\partial C}{\partial a_{1}}=\frac{C}{\alpha_{1}} \frac{\partial \alpha_{1}}{\partial a_{1}}+\frac{C}{\alpha_{2}} \frac{\partial \alpha_{2}}{\partial a_{1}}+\ldots \\
& \frac{\partial C}{\partial a_{2}}=\frac{C}{\alpha_{1}} \frac{\partial \alpha_{1}}{\partial a_{2}}+\frac{C}{\alpha_{2}} \frac{\partial \alpha_{2}}{\partial a_{2}}+\ldots \\
& \therefore \quad\left(a_{k} \frac{\partial}{\partial a_{1}}+a_{k+1} \frac{\partial}{\partial a_{2}}+\ldots a_{k-1} \frac{\partial}{\partial a_{n}}\right) C=C\left(1+\theta+\theta^{2}+\ldots \theta^{n-1}\right)=0 \\
& \text { (13) or } \Sigma a_{k} \frac{\partial C}{\partial a_{1}}=0, k \neq 1 \text {. }
\end{aligned}
$$

By Euler's theorem for homogeneous functions we have

$$
\begin{aligned}
& \Sigma a_{1} \frac{\partial}{\partial a_{1}} C=n C, \text { and } \\
& \left(\Sigma a_{1} \frac{\partial}{\partial a_{1}}\right) \Sigma A_{1}=(n-1) \Sigma A_{1}, \\
& \left(\Sigma a_{1} \frac{\partial}{\partial a_{1}}\right) A_{k}=(n-1) A_{k} .
\end{aligned}
$$

7. It is readily seen that

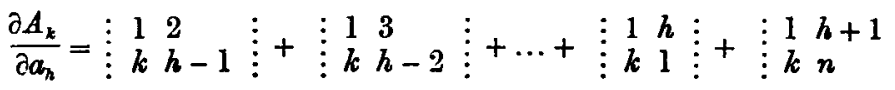

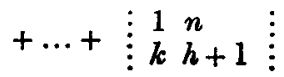

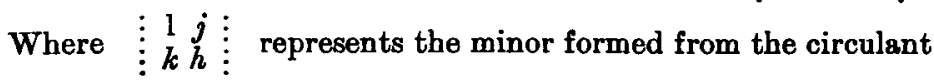
by deleting the $1^{\text {th }}$ and $j^{\text {th }}$ rows and the $k^{\text {th }}$ and $h^{\text {th }}$ columns,

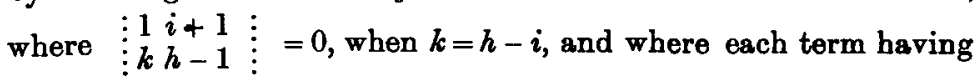
an odd number of inversions is to have a negative sign.

Since for circulants any minor

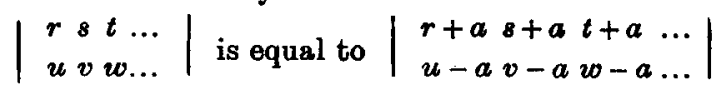


where when any row number becomes greater than $n$ it must be reduced by $n$, and where any column number that becomes zero or negative must be increased by $n$, it is seen that for circulants of even order $n=2 m$ we have

$$
A_{k}=A_{2 k-1}=A_{\substack{m+k \\ m+k}}(k=1,2, \ldots m) \text {, and therefore the primary }
$$

minors corresponding to elements in the odd places of the first row have primary minors corresponding to elements along the principal diagonal equal to them, but those in the even places have not. It follows that $\Sigma A_{11}=2 \Sigma A_{2 k-1}(k=1,2 \ldots m)$, but $\Sigma A_{1}=\Sigma A_{2 k-1}+\Sigma A_{2 k}$,

$$
\Sigma_{i} \frac{\partial}{\partial a_{i}} \Sigma A_{1}=0, \text { and } \Sigma_{i} \frac{\partial}{\partial a_{i}} \Sigma_{A_{11}}=n \frac{\Sigma A_{11}}{\Sigma a_{11}} \text {, }
$$

(17) $\therefore \quad \Sigma_{i} \frac{\partial}{\partial a_{i}} \Sigma A_{2 k-1}=-\Sigma i \frac{\partial}{\partial a_{i}} \Sigma_{A_{2 k}}$

(18) and $\Sigma i \frac{\partial}{\partial a_{i}} \Sigma A_{2 k-1}=\frac{n}{2} \frac{\Sigma A_{11}}{\Sigma a_{11}}$.

In this case of circulants of odd order for every signed primary minor corresponding to elements in the first row there is a primary minor corresponding to some elements along the principal diagonal.

8. If we denote $a_{k} \frac{\partial}{\partial a_{1}}+a_{k+1} \frac{\partial}{\partial a_{2}}+\ldots+a_{n} \frac{\hat{o}}{\partial a_{n-k+1}}$

$$
+a_{1} \frac{\partial}{\partial a_{n-k+2}}+\ldots+a_{k-1} \frac{\partial}{\partial a_{n}}
$$

by $\Sigma a_{k} \frac{\partial}{\partial a_{1}}$, then

$$
\begin{gathered}
\left(\Sigma a_{k} \frac{\partial}{\partial a_{1}}\right) A_{h}=-A_{n-k+1},\left(\text { or } A_{n+h-k+1} \text { if } k>h\right) \\
\left\{\begin{array}{l}
h=1,2, \ldots n \\
k=2,3, \ldots n
\end{array}\right\}
\end{gathered}
$$

The truth of this is seen on observing that $\left(\sum a_{k} \frac{\partial}{\partial a_{1}}\right) A_{h}$ is the sum of $n$ determinants which are obtained by increasing the subscripts of the elements in the first, second, and so on, columns of $A_{n}$ by $k-1$. Of these all vanish, having identical columns, except the $(h-k+1)^{\text {th }}$ (or the $\overline{n+h-k^{\text {th }}}$ if $h<k$ ), and it is $-A_{n-k+1}$ (or $-A_{n+n-k-1}$ ). 
If $h=k$ then (16) becomes

$$
\left(\Sigma a_{k} \frac{\partial}{\partial a_{1}}\right) A_{k}=-A_{1},(k=2,3 \ldots n) .
$$

From the foregoing we have

$$
\begin{aligned}
& \left(\Sigma o_{k} \frac{\partial}{\partial a_{1}}\right) \Sigma A_{1}=-\Sigma A_{1} \text {, or it may be seen as follows : } \\
& C=\Sigma a_{1} \Sigma A_{1} \\
& a_{k} \frac{\partial}{\partial a_{1}} C=a_{k} \Sigma A_{1}+\Sigma a_{1}\left(a_{k} \frac{\partial}{\partial a_{1}} \Sigma A_{1}\right) \\
& a_{k+1} \frac{\partial}{\partial a_{2}} C=a_{k+1} \Sigma A_{1}+\Sigma a_{1}\left(a_{k+1} \frac{\partial}{\partial a_{2}} \Sigma A_{1}\right) \\
& \therefore \quad\left(\Sigma a_{k} \frac{\partial}{\partial a_{1}}\right) C=\Sigma a_{1} \Sigma A_{1}+\Sigma a_{1}\left(\Sigma a_{k} \frac{\partial}{\partial a_{1}} \Sigma A_{1}\right),
\end{aligned}
$$

or $\quad \Sigma a_{k} \frac{\partial}{\partial a_{1}} \Sigma A_{1}=-\Sigma A_{1}$, since $\Sigma a_{k} \frac{\partial}{\partial a_{1}} C=0$.

From $\frac{\partial C}{\partial a_{k}}=n A_{k}$ we get

$$
a_{1} \frac{\partial^{2} C}{\partial a_{k} \partial a_{1}}=n a_{1} \frac{\partial A_{k}}{\partial a_{1}}, a_{2} \frac{\partial^{2} C}{\partial a_{k} \partial a_{2}}=n a_{2} \frac{\partial A_{k}}{\partial a_{2}}, \ldots
$$

and therefore

$$
\begin{aligned}
a_{1} \frac{\partial^{2} C}{\partial a_{k} \partial a_{1}}+a_{2} \frac{\partial^{2} C}{\partial a_{k} \partial a_{2}}+\ldots=n\left(a_{1} \frac{\partial}{\partial a_{1}}+a_{2} \frac{\partial}{\partial a_{2}}+\ldots\right) & A_{k} \\
& \approx n(n-1) A_{k}
\end{aligned}
$$

Also

$$
\begin{aligned}
a_{k} \frac{\partial^{2} C}{\partial a_{k} \partial a_{1}}+a_{k+1} \frac{\partial^{2} C}{\partial a_{k} \partial a_{2}}+\ldots & =n\left(a_{k} \frac{\partial}{\partial a_{1}}+a_{k+1} \frac{\partial}{\partial a_{2}}+\ldots\right) A_{k} \\
& =-n A_{1} .
\end{aligned}
$$

Again, from $C=\Sigma a_{1} \Sigma A_{1}$ we get

$$
\begin{aligned}
& a_{1} \frac{\partial^{2} C}{\partial a_{1}{ }^{2}}=2 a_{1} \frac{\partial}{\partial a_{1}} \Sigma A_{1}+\left(\Sigma a_{1}\right)\left(a_{1} \frac{\partial^{2}}{\partial a_{1}{ }^{2}} \Sigma A_{1}\right), \\
& a_{2} \frac{\partial^{2} C}{\partial a_{1} \partial a_{2}}=a_{2} \frac{\partial}{\partial a_{2}} \Sigma A_{2}+a_{2}\left(\frac{\partial}{\partial a_{1}} \Sigma A_{1}\right)+\left(\Sigma a_{1}\right)\left(a_{2} \frac{\partial^{2}}{\partial a_{1} \partial a_{2}} \Sigma A_{1}\right)
\end{aligned}
$$

and therefore

$$
\begin{aligned}
\left(a_{1} \frac{\partial^{2}}{\partial a_{1}^{2}}+a_{2}\right. & \left.\frac{\delta^{2}}{\partial a_{1} \partial a_{2}}+a_{3} \frac{\partial^{2}}{\partial a_{1} \partial a_{3}}+\ldots\right) C=\left(\Sigma a_{1} \frac{\partial}{\partial a_{1}}\right) \Sigma A_{1} \\
& +\Sigma a_{1}\left(\frac{\partial}{\partial a_{1}} \Sigma A_{\mathrm{r}}\right)+\Sigma a_{1}\left(a_{1} \frac{\partial^{2}}{\partial a_{1}^{2}}+a_{2} \frac{\partial^{2}}{\partial a_{1} \partial a_{2}} \ldots\right) \Sigma A_{1}
\end{aligned}
$$


or

$$
\begin{aligned}
n(n-1) A_{1}=(n-1) \Sigma A_{1} & +\left(n A_{1}-\Sigma A_{1}\right) \\
& +\Sigma a_{1}\left(a_{1} \frac{\partial^{2}}{\partial a_{1}{ }^{2}}+a_{2} \frac{\partial^{2}}{\partial a_{1} \partial a_{2}} \ldots\right) \Sigma A_{1},
\end{aligned}
$$

(23) $\therefore \quad \Sigma a_{1}\left(a_{1} \frac{\partial^{2}}{\partial a_{1}^{2}}+a_{2} \frac{\partial^{2}}{\partial a_{1} \partial a_{2}}+\ldots\right) \Sigma A_{1}=(n-2)\left(n A_{1}-\Sigma A_{1}\right)$,

and also

$$
\left(\Sigma a_{1} \frac{\partial^{2}}{\partial a_{1}^{2}}\right) C=\Sigma a_{1}\left(\Sigma a_{1} \frac{\partial^{2}}{\partial a_{1}^{2}}\right) \Sigma A_{1}+2(n-1) \Sigma A_{1}
$$

and

(25) $\Sigma a_{1}\left(a_{k} \frac{\partial^{2}}{\partial a_{k} \partial a_{1}}+a_{k+1} \frac{\partial^{2}}{\partial a_{k} \partial a_{2}}+\ldots\right) \Sigma A_{1}=2 \Sigma A_{1}-n\left(A_{1}+A_{k}\right)$

\section{From}

$\Sigma_{i} \frac{\partial^{2}}{\partial a_{k} \partial a_{i}} C=n\left(\frac{\partial}{\partial a_{k}} \Sigma A_{1}\right)=n \frac{\partial}{\partial a_{k}} \Sigma A_{1}+\Sigma a_{1}\left(\Sigma i \frac{\partial^{2}}{\partial a_{k} \partial a_{i}} \Sigma A_{i}\right)$ $+\Sigma i \frac{\partial}{\partial a_{i}} \Sigma A_{1}$

and

$$
\Sigma_{i} \frac{\partial^{2}}{\partial a_{k} \partial a_{1}} C=n\left(\Sigma i \frac{\partial}{\partial a_{i}}\right) A_{k}
$$

it follows that

(26) $\frac{\partial}{\partial a_{k}} \Sigma A_{1}=\left(\Sigma i \frac{\partial}{\partial a_{i}}\right) A_{k}$, and

(27) $\Sigma i \frac{\partial^{2}}{\partial a_{k} \partial a_{i}} \Sigma A_{1}=0$, since $\Sigma i \frac{\partial}{\partial a_{i}} \Sigma A_{1}=0$.

10. Another form for writing the circulant is

and therefore

$$
C=a_{1} A_{1}+a_{2} A_{2}+\ldots+a_{n} A_{n} \text {, }
$$

$$
\frac{\partial C}{\partial a_{k}}=a_{1} \frac{\partial A_{1}}{\partial a_{k}}+a_{2} \frac{\partial A_{2}}{\partial a_{k}}+\ldots+a_{k} \frac{\partial A_{k}}{\partial a_{k}} A_{k}+\ldots+a_{n} \frac{\partial A_{n}}{\partial a_{k}} .
$$

(28) or $\Sigma a_{i} \frac{\partial}{\delta a_{k}} A_{i}=(n-1) A_{k}$

Also

(29) $\quad \sum_{i} a_{i} \frac{\partial^{2}}{\partial a_{k}^{2}} A_{i}=(n-2) \frac{\partial}{\partial a_{k}} A_{k}$

(30) $\quad \therefore \quad n \sum i a_{i} \frac{\partial^{2}}{\partial a_{k}^{2}} A_{i}=(n-2) \frac{\partial^{2} C}{\partial a_{k}^{2}}$. 
Taking the sum of both sides of (28) with respect to $k$ gives $a_{1}\left(\frac{\partial}{\partial a_{1}}+\frac{\partial}{\partial a_{2}}+\ldots\right) A_{1}+a_{2}\left(\frac{\partial}{\partial a_{1}}+\frac{\partial}{\partial a_{2}}+\ldots\right) A_{2}+\ldots=(n-1) \sum A_{1}$

(31) or $\Sigma i a_{i} \Sigma x \frac{\partial}{\partial a_{k}} A_{i}=(n-1) \Sigma A_{1}$,

and therefore

$$
\sum_{i} a_{i} \Sigma_{k} \frac{\partial}{\partial a_{k}} A_{1}=\left(\Sigma a_{1} \frac{\partial}{\partial a_{1}}\right) \Sigma A_{1} .
$$

11. As an illustration of some of the foregoing relations let us find the value of the Hessian of a circulant. Taking for convenience sake, the case when the order is five, though the process is obviously general, we have

$H(C) . C$

$$
\begin{aligned}
& =\left|\begin{array}{lllll}
\frac{\partial^{2} C}{\partial a_{1}^{2}} & \frac{\partial^{2} C}{\partial a_{1} \partial a_{2}} & \frac{\partial^{2} C}{\partial a_{1} \partial a_{3}} & \frac{\partial^{2} C}{\partial a_{1} \partial a_{4}} & \frac{\partial^{2} C}{\partial a_{1} \partial a_{5}} \\
\frac{\partial^{2} C}{\partial a_{2} \partial a_{1}} & \frac{\partial^{2} C}{\partial a_{2}^{2}} & \frac{\partial^{2} C}{\partial a_{2} \partial a_{3}} & \frac{\partial^{2} C}{\partial a_{2} \partial a_{4}} & \frac{\partial^{2} C}{\partial a_{2} \partial a_{5}} \\
\frac{\partial^{2} C}{\partial a_{3} \partial a_{1}} & \frac{\partial^{2} C}{\partial a_{3} \partial a_{2}} & \frac{\partial^{2} C}{\partial a_{3}^{2}} & \frac{\partial^{2} C}{\partial a_{4} \partial a_{4}} & \frac{\partial^{2} C}{\partial a_{8} \partial a_{5}} \\
\frac{\partial^{2} C}{\partial a_{4} \partial a_{1}} & \frac{\partial^{2} C}{\partial a_{4} \partial a_{2}} & \frac{\partial^{2} C}{\partial a_{4} \partial a_{3}} & \frac{\partial^{2} C}{\partial a_{4}^{2}} & \frac{\partial^{2} C}{\partial a_{4} \partial a_{5}} \\
\frac{\partial^{3} C}{\partial a_{5} \partial a_{1}} & \frac{\partial^{2} C}{\partial a^{5} \partial a_{2}} & \frac{\partial^{2} C}{\partial a_{5} \partial a_{3}} & \frac{\partial^{2} C}{\partial a_{5} \partial a_{4}} & \frac{\partial^{2} C}{\partial a_{2}^{6}}
\end{array}\right|\left|\begin{array}{lllll}
a_{3} & a_{2} & a_{3} & a_{4} & a_{5} \\
a_{2} & a_{3} & a_{4} & a_{5} & a_{1} \\
a_{3} & a_{4} & a_{5} & a_{1} & a_{2} \\
a_{4} & a_{5} & a_{1} & a_{2} & a_{3} \\
a_{5} & a_{1} & a_{2} & a_{3} & a_{4}
\end{array}\right| \\
& =\mid \begin{array}{rrrrr}
n(n-1) A_{1} & n(n-1) A_{2} & n(n-1) A_{3} & n(n-1) A_{4} & n(n-1) A_{5} \\
-n A_{5} & -n A_{1} & -n A_{2} & -n A_{3} & -n A_{4} \\
-n A_{4} & -n A_{5} & -n A_{1} & -n A_{2} & -n A_{3} \\
-n A_{3} & -n A_{4} & -n A_{5} & -n A_{1} & -n A_{2} \\
-n A_{2} & -n A_{3} & -n A_{4} & -n A_{5} & -n A_{1}
\end{array} \\
& =(-1)^{\frac{1}{2}(n+1)(n-2)}(n-1) n^{n} C^{n-1} \text {. }
\end{aligned}
$$

Therefore

$$
H(C)=(-1)^{\frac{1}{(n+1)(n-2)}(n-1) n^{n} C^{n-2}} .
$$

